\title{
Differential impact of local stiffening and narrowing on hemodynamics in repaired aortic coarctation: an FSI study
}

Liesbeth Taelman ${ }^{1}$, Joris Bols ${ }^{2}$, Joris Degroote ${ }^{2}$, Vivek Muthurangu ${ }^{3}$, Joseph Panzer ${ }^{4}$, Jan Vierendeels $^{2}$, Patrick Segers ${ }^{1}$

${ }^{1}$ IBiTech-bioMMeda, iMinds Medical IT, Faculty of Engineering and Architecture, Ghent University, De Pintelaan 185, 9000 Gent, Belgium

${ }^{2}$ Department of Flow, Heat and Combustion Mechanics, Faculty of Engineering and Architecture, Ghent University, Sint-Pietersnieuwstraat 41, 9000 Gent, Belgium

${ }^{3}$ Centre for Cardiovascular MR, UCL Institute of Child Health, London Great Ormond Street Hospital for Children, Great Ormond Street, London WC1N 3JH

${ }^{4}$ Paediatric Cardiology, Ghent University Hospital, De Pintelaan 185, 9000 Gent, Belgium

Number of words of the manuscript: 6147, of the abstract: 198, of the appendix: 1140

Number of figures: 7

Corresponding author: Patrick Segers

$\mathrm{T}:+3293324198$

Address: De Pintelaan 185, 9000 Gent, Belgium

Email address: Patrick.Segers@UGent.be 


\begin{abstract}
Even after successful treatment of aortic coarctation, a high risk of cardiovascular morbidity and mortality remains. Uncertainty exists on the factors contributing to this increased risk among which the presence of (1) a residual narrowing, leading to an additional resistance and (2) a less distensible zone disturbing the buffer function of the aorta. As the many interfering factors and adaptive physiologic mechanisms present in vivo prohibit the study of the isolated impact of these individual factors, a numerical fluid-structure interaction model is developed to predict central hemodynamics in coarctation treatment. The overall impact of a stiffening on the hemodynamics is limited, with a small increase in systolic pressure (up to $8 \mathrm{mmHg}$ ) proximal to the stiffening which is amplified with increasing stiffening and length. A residual narrowing, on the other hand, affects the hemodynamics significantly. For a short segment (10mm) the combination of a stiffening and narrowing (coarctation index 0.5 ) causes an increase in systolic pressure of $58 \mathrm{mmHg}$, with $31 \mathrm{mmHg}$ due to narrowing and an additional $27 \mathrm{mmHg}$ due to stiffening. For a longer segment $(25 \mathrm{~mm})$, an increase in systolic pressure of $50 \mathrm{mmHg}$ is found, of which only $9 \mathrm{mmHg}$ is due to stiffening.
\end{abstract}

Keywords: Fluid-structure interaction, Stent, End-to-end anastomosis, Image-based modeling 


\section{Introduction}

Aortic coarctation $(\mathrm{CoA})$ is a congenital disease, characterized by a narrowing of the upper descending aorta, obstructing the blood flow from the heart towards the lower part of the body. The treatment can be minimally invasive using a stent and/or a balloon catheter to dilate the coarctation zone, or the narrow section can be removed surgically. Even after a successful treatment, a high risk of cardiovascular morbidity and mortality remains with a.o. recoarctation, aortic aneurysm formation or aortic dissection, left ventricular hypertrophy, premature coronary atherosclerosis, cerebrovascular accidents and systemic hypertension $[32,43]$. This suggests surgical or transcatheter treatments modify rather than correct the complex pathology of aortic coarctation $[4,9,29]$ and coarctation cannot be considered an uncomplicated disease.

In 1971 O'Rourke [31] first related morbidity in CoA (repair) to adverse hemodynamics and biomechanics in the thoracic aorta and the side branches. Considering disturbed blood flow strongly affects vascular pathogenesis, and vice versa, hemodynamic information is of high clinical importance, amongst others to diagnose cardiovascular malfunctioning and evaluate treatment outcomes. However, the majority of the clinical CoA studies focuses on the prevalence of cardiovascular complications $[7,17,37]$, the rates of mortality $[7,17,35]$ and the postinterventional relief of the pressure drop across the coarctation zone $[2,13,18]$ rather than the correlation of hemodynamic indices with manifestation of late morbidity. As such, the underlying role of hemodynamics in the progression of the disease is currently not well understood. With advances in computing power, clinical imaging and segmentation software, computational simulations are nowadays an optimal tool to study the patient specific 
hemodynamics and/or the biomechanics in (repaired) $\mathrm{CoA}$, as they can retrieve data that are difficult to obtain in vivo.

Uncertainty exists on the factors contributing to the increased morbidity among others the presence of a residual narrowing (recurrent coarctation) and a less distensible zone, caused by the presence of a stent or scar tissue. As approximately $60 \%$ of the buffer capacity of the aorta is located in the proximal aorta [36], this local stiffening affects the 'cushioning' function of the aorta. The local narrowing, on the other hand, leads to an additional resistance in the arterial system. In addition, a local narrowing and stiffening generates wave reflections that reach the heart fast, given the short distance to the heart [40].

The many interfering factors and adaptive physiologic mechanisms present in vivo prohibit the study of the isolated impact of these individual factors. As experimental or computational studies more easily allow to mimic the alterations caused by a single parameter, these approaches are crucial in the understanding of central aortic hemodynamics following coarctation treatment. Although there is considerable literature on computational modeling of aortic coarctation, most studies do not account for the elasticity of the aorta and the fluid-structure interaction (FSI) $[19,22,30,39,44]$ and/or have their focus on the hemodynamic impact of coarctation in patientspecific cases [20] or on arterial wall stress and remodeling [8]. The aim of this work is to develop a physiologically relevant 3D model of the aorta with a parametric model for the coarctation zone to predict the hemodynamic impact of (coexisting) stiffening and narrowing in CoA repair. Varying lengths, stiffnesses and diameter reductions of the coarctation zone are studied using fluid-structure interaction simulations and the results are compared against the reference case of the healthy subject. 


\section{Methods}

To obtain the geometric model of the aorta, MR images of a healthy 39 years old male volunteer were taken. The protocol was approved by UK's national research ethics committee and written informed consent was obtained from the volunteer. Semi-automatic segmentation (Mimics, Materialise) resulted in a 3D reconstruction of the aortic arch and thoracic aorta. At the boundaries of the computational domain, the flow rates were measured with phase-contrast MRI and processed using Osirix. The PCMR data was encoded in one direction (through plane) to acquire volumetric flow with the encoding velocities optimized to the peak through plane velocity in the vessel of interest. These measured flow waveforms are imposed as a boundary condition at the ascending aorta and the three side branches (see Figure 1) assuming a flat velocity profile. Mean aortic inflow was $129.8 \mathrm{ml} / \mathrm{s}$, while mean outflow via the right brachiocephalic, left common carotid and left subclavian artery was 18.9, 7.6 and $15.9 \mathrm{ml} / \mathrm{s}$, respectively. At the descending aorta, a 3-element windkessel model is implemented, for which the parameters are defined such that physiological pressure variations are retrieved $\left(Z_{c}=0.08\right.$ $\mathrm{mmHg} /(\mathrm{ml} / \mathrm{s}), R=1.024 \mathrm{mmHg} /(\mathrm{ml} / \mathrm{s}), C=2.0 \mathrm{ml} / \mathrm{mmHg})$. These values were obtained by fitting a 3-element windkessel model to the data, imposing the measured descending aorta flow as input and minimizing the difference between model-predicted pressure and a "measured" pressure waveform. The latter was generated by using the descending aortic distension waveform as a substitute for the pressure waveform and scaling it to the measured brachial diastolic ( 80 $\mathrm{mmHg})$ and systolic $(115 \mathrm{mmHg})$ blood pressure. The subject's heart rate was 64 beats/minute.

As computational fluid dynamics (CFD) simulations with rigid walls fail to capture some physiological patterns (such as wave propagation and reflection), the fluid-structure interaction 
(FSI) between the blood flow and the deformation of the arterial wall is taken into account. The governing equations for the blood flow and the deformation of the structure are solved with two separate codes (Ansys, Fluent and Simulia, Abaqus/Standard resp.), which are strongly coupled. This approach allows the flow equations and the structural equations to be solved with different techniques that are particularly suited to solve the respective equations. In this work a quasiNewton algorithm with an approximation for the inverse of the Jacobian (IQN-ILS) is used to solve the coupled problem [11]. This algorithm influences only the interface displacement, all remaining variables in the fluid and solid domain are considered as internal variables. It thereby treats both the flow and the structural solver as a black box which allows the use of commercial software packages. In [11] the IQN-ILS technique is compared with other partitioned schemes, such as Aitken relaxation and Interface-GMRES(R). This comparison indicates that fewer coupling iterations per time step are required if the IQN-ILS algorithm is used. To obtain an accurate calculation of the stress on the fluid-structure interface, the flow equations are solved in the Arbitrary Lagrangian-Eulerian formulation on a deforming mesh.

To create a geometric model of the aortic tissue, the aortic lumen was extended such that a diameter to thickness ratio of $10 \%$ was obtained. At the boundaries, only radial displacement is allowed. The material behavior of the aortic tissue is described using a polynomial hyperelastic model (hyperelastic constants: $C_{10}=18.9 \mathrm{kPa}, C_{01}=2.75 \mathrm{kPa}, C_{20}=400 \mathrm{kPa}, C_{11}=847.2 \mathrm{kPa}$ [34]) with the value of $\mathrm{C}_{20}$ obtained in an iterative way, such that the deformations of the descending aorta in the FSI simulation corresponded to the deformations measured with MRI $(9 \%)$. 
Using the extended Treemesh method [1] an automated high quality hexahedral mesh was generated both in the fluid and solid domain. Hexahedral meshes are superior to tetrahedral/prismatic meshes as they converge better, and require less computational time for the same accuracy [10]. This method furthermore allowed to create an additional refinement in the flow region distal to the coarctation where vortices develop. A mesh sensitivity study eventually led to a grid with $216 \mathrm{k}$ and $51 \mathrm{k}$ linear cells in the fluid and solid domain respectively. A time step size of $2 \mathrm{~ms}$ is used to resolve the flow field in time. Details on the mesh and time step sensitivity study are provided in Appendix 1 . The structural model, the flow model and the interaction between both, allow to predict the central hemodynamics in a healthy aorta. These results will be used as a reference for the other simulations.

To model the functional impact of repaired CoA, a segment with varying length $(L)$, stiffness $\left(E_{C O A}\right)$ and diameter $\left(D_{C o A}\right)$ is included (indicated by the colored zone in Figure 2) using the software 3-matic (Materialise). Two types of intervention are considered. (1) Resection by endto-end anastomosis, resulting in circular scar tissue at the location where both ends of the aorta are sutured together. In [41] local elasticity properties of the aortic wall (such as the elasticity modulus $E$ and stiffness-index $\beta$ ) indicate local increase in stiffness in the region of the surgical scar. Based on the stiffness indices and dimensions reported in this article, the elasticity modulus of the coarctation region in our model $\left(E_{C O A}\right)$ is chosen to be equal to 5 or 20 times stiffer than the unaffected aortic tissue $\left(E_{A o}\right)$. A length $(L)$ of, alternately, 10 and $25 \mathrm{~mm}$ is thereby selected (see top left of Figure 2). (2) Relief of the obstruction by stent deployment. In [12], a review on different stent types used in coarctation treatment is given and considerations to achieve successful stent implantation are discussed. The segment lengths applied in our research (20 and 
$50 \mathrm{~mm}$ ) are chosen to cover the range of stent lengths currently used in coarctation repair. The presence of the noncompliant stent increases aortic stiffness. We arbitrarily assumed a 100 times stiffer material to mimic the wall behavior in the stented section (see top right of Figure 2).

The severity of the residual stenosis is quantified by the coarctation index $(C I)$, defined as the ratio of the diameter of the coarctation zone to the diameter of the descending aorta $\left(D_{C o A} / D_{D A o}\right)$. The higher the $C I$, the lower the severity of the recurrent narrowing. Two gradations of severity are considered: an index of 0.5 indicating a severe stenosis which requires treatment and an index of 0.65 , mimicking a mild narrowing, which does not necessitate intervention [3] (see Figure 2 bottom).

\section{Impact of rigid wall modeling}

In a first study the impact of a rigid wall assumption in the assessment of coarctation severity is considered. For this purpose both a rigid wall (CFD) and a flexible wall (FSI) simulation are performed for the case of a severe stenosis $\left(L=25 \mathrm{~mm}, E_{C o A}=E_{A o}\right.$ and $\left.C I=0.5\right)$. The aortic geometry used in the CFD study corresponds to the one extracted from MR images. The same boundary conditions were used in both simulations.

\section{Impact of repaired CoA}

Next, the effect of repaired aortic coarctation on the central hemodynamics is studied for the parameter models shown in Figure 2. In particular the pressure at the ascending aorta, the pressure drop across the coarctation region and the flow patterns are discussed. 


\section{Results}

\section{Impact of rigid wall modeling}

Figure 3 compares the pressure drop and the velocity along the centerline of the aorta obtained with a rigid wall and a flexible wall simulation. For the rigid wall simulation results are shown at peak systole. For the FSI simulation, the red curve is obtained at peak systolic inflow $(\mathrm{t}=0.094$ s), whereas the black curve is retrieved at the time point where the flow at the constriction site and thus, the pressure gradient becomes maximal $(\mathrm{t}=0.174 \mathrm{~s})$. The CFD simulation strongly overestimates CoA severity as both the pressure drop across the coarctation (101 versus 57 $\mathrm{mmHg}$ ) and the pressure difference between the ascending and descending aorta (62 versus 44 $\mathrm{mmHg}$ ) are amplified.

\section{Impact of repaired CoA on proximal pressure}

Figure 4 depicts the impact of a local stiffening and/or narrowing on the pressure at the ascending aorta (i.e. the inlet of the model), averaged over the cross section. In the top left panel, the effect of an isolated stiffening is shown for the two worst cases: circular scar tissue with a length of $25 \mathrm{~mm}$ (20 times stiffer than the unaffected aortic tissue; black curve) and a $50 \mathrm{~mm}$ long stent (red curve). Only a small pressure build-up around peak systole is found, rising up to 8 mmHg with increasing stiffening and length.

The effect of a residual narrowing is illustrated in the right top panel of Figure 4. Here, a more pronounced impact, covering the whole systolic phase is observed. A coarctation index of 0.65 increases peak systolic pressure by $10 \mathrm{mmHg}$ (red and green curve), independent of the length of the coarctation zone, whereas an index of 0.5 elevates the load on the heart up to 31 and 41 
$\mathrm{mmHg}$ for a segment with a length of 10 (black curve) or $25 \mathrm{~mm}$ (magenta curve) respectively.

The combined effect of a narrowing $(C I=0.5)$ and a stiffening is shown in the charts at the bottom of Figure 4. For a short segment $(L=10 \mathrm{~mm})$ an additional stiffening will have a significant impact on the pressure evolution (up to $27 \mathrm{mmHg}$ ) whereas the impact of stiffening for a longer segment is relatively limited (up to $9 \mathrm{mmHg}$ ).

\section{Impact of repaired CoA on pressure drop}

The time-averaged pressure along the aorta is depicted in Figure 5, in which the location of the coarctation segment is indicated by the colored zone. In a normal aorta, the mean pressure decrease is limited to $0.8 \mathrm{mmHg}$. The inclusion of a local stiffening hardly affects this pressure reduction. A sharp fall of the mean pressure near the constriction is retrieved if a narrowing is present. For a coarctation index of 0.65 , the mean pressure reduces by $3.8 \mathrm{mmHg}$ across the stenosis. This value further increases up to 9.1 and $11.3 \mathrm{mmHg}$ for a more severe stenosis degree with a length of $10 \mathrm{~mm}$ and $25 \mathrm{~mm}$ respectively. This pressure drop is accompanied by pressure recovery, persisting through a larger part of the distal aorta. Severe coarctation $(C I=0.5)$ is characterized by a smaller pressure recovery of $25 \%$ and $21 \%$ for a length of 10 and $25 \mathrm{~mm}$, whereas mild coarctation results in a recovery of $31 \%$.

The comparison of the pressure distribution along the aorta between the reference case and the most severe case of repaired $\mathrm{CoA}\left(L=10 \mathrm{~mm}, E_{C o A}=20 E_{A o}\right.$ and $\left.C I=0.5\right)$ is made in Figure 6 at the time of maximal instantaneous pressure drop $\left(\Delta p_{\max }\right)$ between the ascending and descending aorta (indicated in blue and red respectively). This pressure difference is also indicated in the 
charts on top of this figure and differs from the peak-to-peak pressure difference $\left(\Delta p_{p t p}\right)$ often reported in literature, which is a nonphysiological measurement as the maxima at the ascending and descending aorta occur at different points in time. This last pressure difference is thus never experienced by the patient. For the normal aorta, a gradual decrease in pressure along the aortic length can be observed. For the case with recurrent coarctation pressures falls sharply as the constriction is approached. At the distal end of the coarctation, flow deceleration is accompanied by pressure recovery which, on the other hand, takes place over the entire descending aorta.

\section{Impact of repaired CoA on the flow patterns}

Figures 6 shows the velocity contour images of the aortic arch at the time point for which the pressure gradient between the ascending and descending aorta becomes maximal. Comparison is made between the reference case and the worst case (in terms of pressure gradient across the CoA zone $)$ of repaired $\mathrm{CoA}\left(C I=0.5, L=10 \mathrm{~mm}, E_{C o A}=20 E_{A o}\right)$. In Figure 7, the corresponding velocity vectors at four locations along the descending aorta are shown. As reported in [19,24], blood acceleration across the coarctation region generates complex flow distal to the stenosis. The shear layer around the flow jet, leaving the coarctation zone, is marked by a rapid growth of instabilities.

Downstream vortices and swirling are produced especially in the deceleration phase in the expansion zone. The maximal velocity increases from a value of $1.07 \mathrm{~m} / \mathrm{s}$ in the normal aorta to a value of $4.7 \mathrm{~m} / \mathrm{s}$ in the repaired CoA and a shift towards the right outer wall is found. Distally, this magnitude decreases and distribution alteration results in a skewed axial velocity profile, characterized by a loss of symmetry and eventually a flow jet impacting on the posterior right outer wall. Compared with the effect of a narrowing, the impact of a local stiffening is thus fairly 
limited. 


\section{Discussion}

\section{Impact of rigid wall modeling}

An important feature of the aorta is its capacity to buffer blood during systole and sustain blood flow to the rest of the body during diastole. As approximately $60 \%$ of the buffer capacity of the healthy aorta is located in the proximal aorta [36], the presence of (repaired) CoA might affect this property. Inclusion of this characteristic hallmark in the numerical model is thus necessary in order to quantify the disease severity correctly. Most CoA studies $[19,21,27,30,39,44]$ are, however, performed under a rigid wall assumption. That this oversimplification may corrupt insights and provide an incorrect diagnosis of CoA severity is illustrated by Figure 3 . This substantial mismatch is related to the lack of compliance in the CFD simulation, which is responsible for the buffering and damping of the pressure pulse in the proximal aorta. Accordingly, velocities in the coarctation zone and the associated pressure drop exceed the ones reported in the FSI simulation. Increased aortic compliance will enhance this buffering effect as it results in an enlarged dilation of the proximal aorta in systole and a further accumulation of stored upstream energy, which is released downstream in diastole. The diminished losses during pressure recovery downstream of the CoA partially compensate the overestimation of the pressure drop across the CoA in the CFD simulation. This might explain the moderate agreement between measured pressure differences and the ones retrieved with rigid wall models [39]. Overall, the results shown in Figure 3 call for a fluid-structure interaction approach in the determination of pressure drops across the CoA using computational models. 


\section{Impact of repaired CoA on proximal pressure}

As reported in [38], in which a physiological pressure pulse was imposed as a boundary condition to a straight, flexible tube including a local stiffening, no significant alteration of the proximal pressure is retrieved. This limited impact is comprehended by the analysis of the wave reflections induced by the stiffening. The backward compression wave generated at the transition from the flexible artery to the rigid segment is roughly canceled out by the expansion waves created at the distal end of the coarctation zone. As such, only local changes in pressure are found, related to the time delay between the backward waves. This finding is in agreement with the $1 \mathrm{D}$ studies performed in $[5,14]$ and the 3D FSI study published in [8], reporting negligible clinical consequences of a local stiffening on cardiac workload and aortic pressure. In [33] an experimental porcine model was developed to investigate the effect of a noncompliant stent. This study supports the conclusions from previous numerical studies.

Regarding the threshold for intervention, a peak-to-peak pressure difference of $20 \mathrm{mmHg}$ between the upper and lower limbs is often used as an intervention criteria. This threshold compromises between the risks and benefits associated with treatment. Patients with mild coarctation are subjected to long-term hypertension or may require lifelong antihypertensive treatment. In addition, the blood pressures emerging during exercise will be much more pronounced than the ones appearing at rest. The success of noninvasive stent implantation in patients with more severe CoA, conjoined with the limited impact on the hemodynamic response may call for a revision of the threshold for intervention [26]. 
For the models with recurrent coarctation, the progressive increase in proximal pressure with coarctation length might be explained by (1) the viscous losses which are proportional to stenosis length and inversely related to the coarctation diameter and (2) the pressure losses due to the unsteady acceleration, which become more pronounced in the stenosis due to the increased velocities and the corresponding accelerations. Remark that the pressure losses due to the unsteady acceleration should manifest in the slope of the pressure curves, as they are present during systole but become negligible around peak systole. As the slope of the pressure curves are more or less equal in all cases, we can conclude that the additional pressure drop due to the unsteady acceleration is very small.

Considering a coexisting stiffening and narrowing, the different response between a short and a long segment might be explained by the difference in wall deformations. In the absence of a local stiffening, the short narrowed segment will experience pronounced deformations up to $29 \%$ as it is pulled apart by the proximal and distal part of the aorta. Conversely, for a long segment, these deformations are restricted to $12 \%$. The smaller cross section in the latter case will result in a higher blood velocity and, as a result, in an elevated pressure drop across the coarctation. For a local stiffening $\left(E_{C o A}=20 E_{A o}\right)$, the distentions of the local narrowing become significantly smaller. A deformation of 3\% is retrieved for the short segment and $2.5 \%$ for the long segment. The difference in pressure evolution in this case can be explained by the difference in shape of the constriction rather than the difference in cross section (which is nearly equal in both cases). The smaller the divergion of the streamlines distal to the stenosis, the better the pressure recovery will be. As such, a smaller pressure difference between the ascending and descending 
aorta is found in the case of a long stiffening which is characterized by a more gradual change in diameter.

\section{Impact of repaired CoA on pressure drop}

For a normal aorta, the dominant factor determining the value of $\Delta p_{\max }$ in the reference case is the inertia of the blood related to the temporal blood acceleration during the systolic phase. In case of recurrent coarctation, the (unsteady) pressure drop adds to a convective acceleration term, caused by an increase in velocity at the transition from the aorta to the stenosis and is proportional to the velocity gradient. This convective acceleration obscures the unsteady acceleration and causes the pressure to fall sharply as the constriction is approached. Distally the conversion of kinetic energy into pressure is accompanied by energy losses related to turbulence development in the descending aorta. These losses together with the viscous losses explain the enlarged pressure drop (up to $36 \mathrm{mmHg}$ ) in case of repaired CoA.

Note that apart from the stenosis severity and geometry, the pressure drop and recovery furthermore depend on flow rate. For exercise conditions, for example, an even more distinctive pressure drop will be found and question remains which pressure difference is most clinically relevant: the one that is present during daily life or the worst case pressure drop, only temporary arising during exercise [6]. This suggests that an assessment of stenosis severity cannot be based on the pressure drop alone, but an additional measurement of blood flow is required. As a second remark, we like to point out that, in this research, a circular symmetrical stiffening and narrowing are applied, mimicking stent implantation or resection by end-to-end anastomosis. Treatment outcomes of other procedures, such as patch aortoplasty or Waldhausen repair will, 
however, result in an asymmetrical stenosis and stiffening. This feature will manifest in an even larger pressure difference across the stenosis and a worsened pressure recovery in the posterior descending aorta [25].

\section{Limitations}

It is import to keep in mind that this is a parametric study where the structural and functional alterations of the coarctation zone were induced in a model entirely based on data obtained from a healthy volunteer. Patients with (repaired) aortic coarctation might have intrinsic structural defects in extracellular matrix proteins due to genetic defects [42], and their aorta has been subjected to growth and remodeling $[16,23]$ with adaptions in shape and material properties. In particular for the case of aortic coarctation, wall thickening is often observed along with a decrease in compliance of the proximal aorta due to prolonged hypertension [8,27]. As such, neither the 3D geometry, neither the assumed material constants in this paper can be considered representative for patients with (repaired) aortic coarctation. This also impacts on the demonstrated differences between the CFD and FSI results, which will be less pronounced when accounting for the reduced distensibility of the proximal aorta in the patient case.

Also, the same boundary conditions were imposed in all cases, regardless of coarctation severity. This approach, however, allows to isolate the hemodynamic alterations caused by the presence of repaired CoA and approximates the autoregulatory mechanisms of the cardiovascular system which keeps the downstream boundary conditions relatively constant. The assumption of a constant cardiac output in CoA implies an increased workload on the heart and is justified by the findings reported in [15], stating that the cardiac output and the heart rate barely change after 
surgically induced stenosis. We, however, believe that, due to the elevated resistance at the coarctation site an early redistribution of flow will take place, manifesting as an increased flow through the subclavian and carotid arteries and a reduction of the descending aortic flow [8]. This was not accounted for in this study. Application of reduced order models at the distal boundaries of the fluid domain might resolve this problem $[8,20]$. Since the pressure drop across the $\mathrm{CoA}$ is proportional to the flow rate through the constriction, the flow distribution adopted in our models represents the worst case distribution, associated with the highest pressure gradients. Similarly, disregarding the collateral network, bypassing part of the flow through the coarctation, will result in an overestimation of the actual pressure drop.

Another limitation of this research involves the lack of (viscoelastic) tissue surrounding the aorta [28]. As such, no physiological mechanism is present to damp the high frequency oscillations of the vessel wall.

\section{Conclusions}

In conclusion, we have used 3D fluid-structure interaction simulations to assess the hemodynamic impact of a narrowing and/or stiffening in an otherwise healthy aorta as a parametric model of (repaired) aortic coarctation. The hemodynamic impact of an isolated stiffening is limited. Aortic constriction, on the other hand, induces a pronounced increase in blood pressure in the proximal aorta, with buffering of the stroke volume proximal to the aortic narrowing. For short constrictions, additional stiffening will have a significant impact on the pressure evolution whereas the impact is relatively limited for longer constricted segments. 
Comparison with CFD simulations highlighted the importance of accounting for the elasticity of the aorta to correctly capture the buffering of the proximal aorta.

\section{Acknowledgments}

This research was funded by the Research Foundation - Flanders (FWO-Vlaanderen), project nr 3G008509.

\section{References}

1. Bols J, De Santis G, Degroote J, Verhegghe B, Segers P, Vierendeels J Automated Hexahedral Mesh Generation in a Complex Vascular Tree: The Extended Treemesh Method. In: ASME 2013 Summer Bioengineering Conference, 2013. American Society of Mechanical Engineers, pp V01AT13A019-V001AT013A019

2. Bouchart F, Dubar A, Tabley A, Litzler PY, Haas-Hubscher C, Redonnet M, Bessou JP, Soyer R (2000) Coarctation of the aorta in adults: Surgical results and long-term follow-up. Annals of Thoracic Surgery 70:1483-1488. doi:10.1016/s0003-4975(00)01999-8

3. Carvalho JS, Redington AN, Shinebourne EA, Rigby ML, Gibson D (1990) Continuous wave doppler echocardiography and coarctation of the aorta - gradients and flow patterns in the assessment of severity. British Heart Journal 64:133-137

4. Celermajer DS, Greaves K (2002) Survivors of coarctation repair: fixed but not cured. Heart 88:113-114. doi:10.1136/heart.88.2.113

5. Charonko JJ, Ragab SA, Vlachos PP (2009) A Scaling Parameter for Predicting Pressure Wave Reflection in Stented Arteries. Journal of Medical Devices-Transactions of the Asme 3. doi:10.1115/1.3089140 
6. Clark C (1976) Fluid-mechanics of aortic-stenosis .2. Unsteady-flow experiments. Journal of Biomechanics 9:567-\&. doi:10.1016/0021-9290(76)90097-x

7. Cohen M, Fuster V, Steele PM, Driscoll D, McGoon DC (1989) Coarctation of the aorta long-term follow-up and prediction of outcome after surgical-correction. Circulation 80:840845

8. Coogan JS, Humphrey JD, Figueroa CA (2013) Computational simulations of hemodynamic changes within thoracic, coronary, and cerebral arteries following early wall remodeling in response to distal aortic coarctation. Biomechanics and Modeling in Mechanobiology 12:7993. doi:10.1007/s10237-012-0383-x

9. de Divitiis M, Rubba P, Calabro R (2005) Arterial hypertension and cardiovascular prognosis after successful repair of aortic coarctation: A clinical model for the study of vascular function. Nutrition Metabolism and Cardiovascular Diseases 15:382-394. doi:10.1016/j.numecd.2005.08.002

10. De Santis G, Mortier P, De Beule M, Segers P, Verdonck P, Verhegghe B (2010) Patientspecific computational fluid dynamics: structured mesh generation from coronary angiography. Medical \& Biological Engineering \& Computing 48:371-380. doi:10.1007/s11517-010-0583-4

11. Degroote J, Bathe K-J, Vierendeels J (2009) Performance of a new partitioned procedure versus a monolithic procedure in fluid-structure interaction. Computers \& Structures 87:793801. doi:10.1016/j.compstruc.2008.11.013

12. Ebeid M (2003) Balloon expandable stents for coarctation of the aorta: review of current status and technical considerations. Images in paediatric cardiology 5:25-41 
13. Fletcher SE, Nihill MR, Grifka RG, Olaughlin MP, Mullins CE (1995) Balloon angioplasty of native coarctation of the aorta - midterm follow-up and prognostic factors. Journal of the American College of Cardiology 25:730-734. doi:10.1016/0735-1097(94)00437-u

14. Formaggia L, Nobile F, Quarteroni A (2002) A one dimensional model for blood flow: Application to vascular prosthesis. In: Ciarlet PG, Miyoshi T (eds) Mathematical Modeling and Numerical Simulation in Continuum Mechanics, vol 19. Lecture Notes in Computational Science and Engineering. pp 137-153

15. Giddens DP, Mabon RF, Cassanova RA (1976) Measurements of disordered flows distal to subtotal vascular stenoses in thoracic aortas of dogs. Circulation Research 39:112-119

16. Humphrey JD (2008) Mechanisms of arterial remodeling in hypertension: coupled roles of wall shear and intramural stress. Hypertension 52:195-200.

doi:10.1161/hypertensionaha.107.103440

17. Jenkins NP, Ward C (1999) Coarctation of the aorta: natural history and outcome after surgical treatment. Qjm-Monthly Journal of the Association of Physicians 92:365-371. doi:10.1093/qjmed/92.7.365

18. Johnston TA, Grifka RG, Jones TK (2004) Endovascular stents for treatment of coarctation of the aorta: Acute results and follow-up experience. Catheterization and Cardiovascular Interventions 62:499-505. doi:10.1002/ccd.20071

19. Keshavarz-Motamed Z, Kadem L (2011) 3D pulsatile flow in a curved tube with coexisting model of aortic stenosis and coarctation of the aorta. Medical Engineering \& Physics 33:315324. doi:10.1016/j.medengphy.2010.10.017

20. Kim HJ, Vignon-Clementel IE, Figueroa CA, LaDisa JF, Jansen KE, Feinstein JA, Taylor CA (2009) On Coupling a Lumped Parameter Heart Model and a Three-Dimensional Finite 
Element Aorta Model. Annals of Biomedical Engineering 37:2153-2169.

doi:10.1007/s10439-009-9760-8

21. LaDisa JF, Jr., Dholakia RJ, Figueroa CA, Vignon-Clementel IE, Chan FP, Samyn MM, Cava JR, Taylor CA, Feinstein JA (2011) Computational Simulations Demonstrate Altered Wall Shear Stress in Aortic Coarctation Patients Treated by Resection with End-to-end Anastomosis. Congenital Heart Disease 6:432-443. doi:10.1111/j.1747-0803.2011.00553.x

22. LaDisa JF, Jr., Figueroa CA, Vignon-Clementel IE, Kim HJ, Xiao N, Ellwein LM, Chan FP, Feinstein JA, Taylor CA (2011) Computational Simulations for Aortic Coarctation: Representative Results From a Sampling of Patients. Journal of Biomechanical EngineeringTransactions of the Asme 133. doi:10.1115/1.4004996

23. Langille BL (1996) Arterial remodeling: Relation to hemodynamics. Canadian Journal of Physiology and Pharmacology 74:834-841. doi:10.1139/cjpp-74-7-834

24. Liu B (2007) The influences of stenosis on the downstream flow pattern in curved arteries. Medical Engineering \& Physics 29:868-876. doi:10.1016/j.medengphy.2006.09.009

25. Marom G, Kim H-S, Rosenfeld M, Raanani E, Haj-Ali R (2013) Fully coupled fluidstructure interaction model of congenital bicuspid aortic valves: effect of asymmetry on hemodynamics. Medical \& Biological Engineering \& Computing 51:839-848. doi:10.1007/s11517-013-1055-4

26. Marshall AC, Perry SB, Keane JF, Lock JE (2000) Early results and medium-term follow-up of stent implantation for mild residual or recurrent aortic coarctation. American Heart Journal 139:1054-1060. doi:10.1067/mhj.2000.106616

27. Menon A, Wendell DC, Wang H, Eddinger TJ, Toth JM, Dholakia RJ, Larsen PM, Jensen ES, LaDisa JF, Jr. (2012) A coupled experimental and computational approach to quantify 
deleterious hemodynamics, vascular alterations, and mechanisms of long-term morbidity in response to aortic coarctation. Journal of Pharmacological and Toxicological Methods 65:1828. doi:10.1016/j.vascn.2011.10.003

28. Moireau P, Xiao N, Astorino M, Figueroa CA, Chapelle D, Taylor CA, Gerbeau JF (2012) External tissue support and fluid-structure simulation in blood flows. Biomechanics and Modeling in Mechanobiology 11:1-18. doi:10.1007/s10237-011-0289-z

29. Oechslin EN (2008) Does a stent cure hypertension? Heart 94:828-829. doi:10.1136/hrt.2007.130013

30. Olivieri LJ, de Zelicourt DA, Haggerty CM, Ratnayaka K, Cross RR, Yoganathan AP (2011) Hemodynamic Modeling of Surgically Repaired Coarctation of the Aorta. Cardiovascular engineering and technology 2:288-295. doi:10.1007/s13239-011-0059-1

31. Orourke MF, Cartmill TB (1971) Influence of aortic coarctation on pulsatile hemodynamics in proximal aorta. Circulation 44:281-\&

32. Pedersen TA (2012) Late morbidity after repair of aortic coarctation. Danish medical journal 59:B4436

33. Pihkala J, Thyagarajan GK, Taylor GP, Nykanen D, Benson LN (2001) The effect of implantation of aortic stents on compliance and blood flow. An experimental study in pigs. Cardiology in the Young 11:173-181. doi:10.1017/s1047951101000075

34. Prendergast PJ, Lally C, Daly S, Reid AJ, Lee TC, Quinn D, Dolan F (2003) Analysis of prolapse in cardiovascular stents: A constitutive equation for vascular tissue and finiteelement modelling. Journal of Biomechanical Engineering-Transactions of the Asme 125:692-699. doi:10.1115/1.1613674 
35. Rothman A (1998) Coarctation of the aorta: An update. Current Problems in Pediatrics 28:37-60. doi:http://dx.doi.org/10.1016/S0045-9380(98)80039-X

36. Stergiopulos N, Segers P, Westerhof N (1999) Use of pulse pressure method for estimating total arterial compliance in vivo. American Journal of Physiology-Heart and Circulatory Physiology 276:H424-H428

37. Stewart AB, Ahmed R, Travill CM, Newman CGH (1993) Coarctation of the aorta life and health 20-44 years after surgical repair. British Heart Journal 69:65-70

38. Taelman L, Degroote J, Swillens A, Vierendeels J, Segers P (2014) Fluid-structure interaction simulation of pulse propagation in arteries: Numerical pitfalls and hemodynamic impact of a local stiffening. International Journal of Engineering Science 77:1-13. doi:10.1016/j.ijengsci.2013.12.002

39. Valverde I, Staicu C, Grotenhuis H, Marzo A, Rhode K, Shi Y, Brown A, Tzifa A, Hussain T, Greil G, Lawford P, Razavi R, Hose R, Beerbaum P (2011) Predicting hemodynamics in native and residual coarctation: preliminary results of a Rigid-Wall Computational-FluidDynamics model (RW-CFD) validated against clinically invasive pressure measures at rest and during pharmacological stress. J Cardiovasc Magn Reson 13:1-4. doi:10.1186/1532429X-13-S1-P49

40. van den Wijngaard JPHM, Siebes M, Westerhof BE (2009) Comparison of arterial waves derived by classical wave separation and wave intensity analysis in a model of aortic coarctation. Medical \& Biological Engineering \& Computing 47:211-220. doi:10.1007/s11517-008-0387-y

41. Verhaaren H, De Mey S, Coomans I, Segers P, De Wolf D, Matthys D, Verdonck P (2001) Fixed region of nondistensibility after coarctation repair: In vitro validation of its influence 
on Doppler peak velocities. Journal of the American Society of Echocardiography 14:580587. doi:10.1067/mje.2001.113256

42. Vogt M, Kühn A, Baumgartner D, Baumgartner C, Busch R, Kostolny M, Hess J (2005) Impaired Elastic Properties of the Ascending Aorta in Newborns Before and Early After Successful Coarctation Repair: Proof of a Systemic Vascular Disease of the Prestenotic Arteries? Circulation 111:3269-3273. doi:10.1161/circulationaha.104.529792

43. Vriend JWJ, Mulder BJM (2005) Late complications in patients after repair of aortic coarctation: implications for management. International Journal of Cardiology 101:399-406. doi:http://dx.doi.org/10.1016/j.ijcard.2004.03.056

44. Wendell DC, Samyn MM, Cava JR, Ellwein LM, Krolikowski MM, Gandy KL, Pelech AN, Shadden SC, LaDisa JF, Jr. (2013) Including aortic valve morphology in computational fluid dynamics simulations: Initial findings and application to aortic coarctation. Medical Engineering \& Physics 35:723-735. doi:10.1016/j.medengphy.2012.07.015 


\section{List of figures}

Fig. 1: Methodological figure with setup and boundary conditions.

Fig. 2: Parameter models of repaired CoA used in this research.

Fig. 3: Comparison of the pressure drop (viewed from the descending aorta) and velocity along the aortic arch centerline obtained with a rigid wall simulation (CFD) and a flexible wall model (FSI) of recurrent coarctation $\left(L=25 \mathrm{~mm}, E_{C o A}=E_{A o}\right.$ and $\left.C I=0.5\right)$. The blue and red curve are obtained at peak systole (i.e. maximal inlet flow), the black curve is generated at a time point for which the pressure drop across the aortic arch reaches its maximum.

Fig. 4: Impact of a local stiffening and/or narrowing on the pressure evolution at the ascending aorta as a function of time.

Fig. 5: Impact of a local stiffening and/or narrowing on the mean pressure evolution as a function of the distance from the model inlet (i.e. the ascending aorta).

Fig. 6: Comparison of (top) the maximal pressure gradient in the reference case and in repaired $\operatorname{CoA}\left(L=10 \mathrm{~mm}, E_{C o A}=20 E_{A o}\right.$ and $\left.C I=0.5\right)$ and (bottom) contourplot of the corresponding velocity magnitude.

Fig. 7: Comparison of the velocity vectors along the descending aorta (planes corresponding to the ones shown in Figure 6) in the reference case and in repaired CoA $\left(L=10 \mathrm{~mm}, E_{C o A}=20\right.$ $E_{A o}$ and $\left.C I=0.5\right)$. 


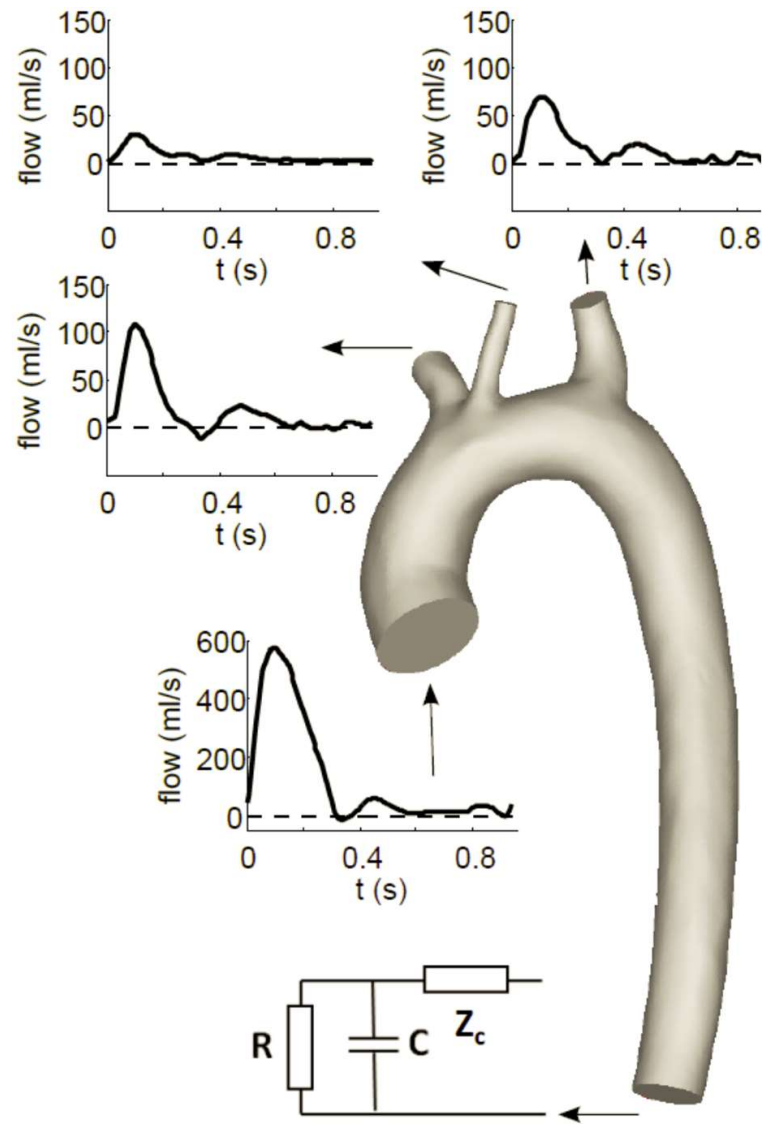

Fig. 1 


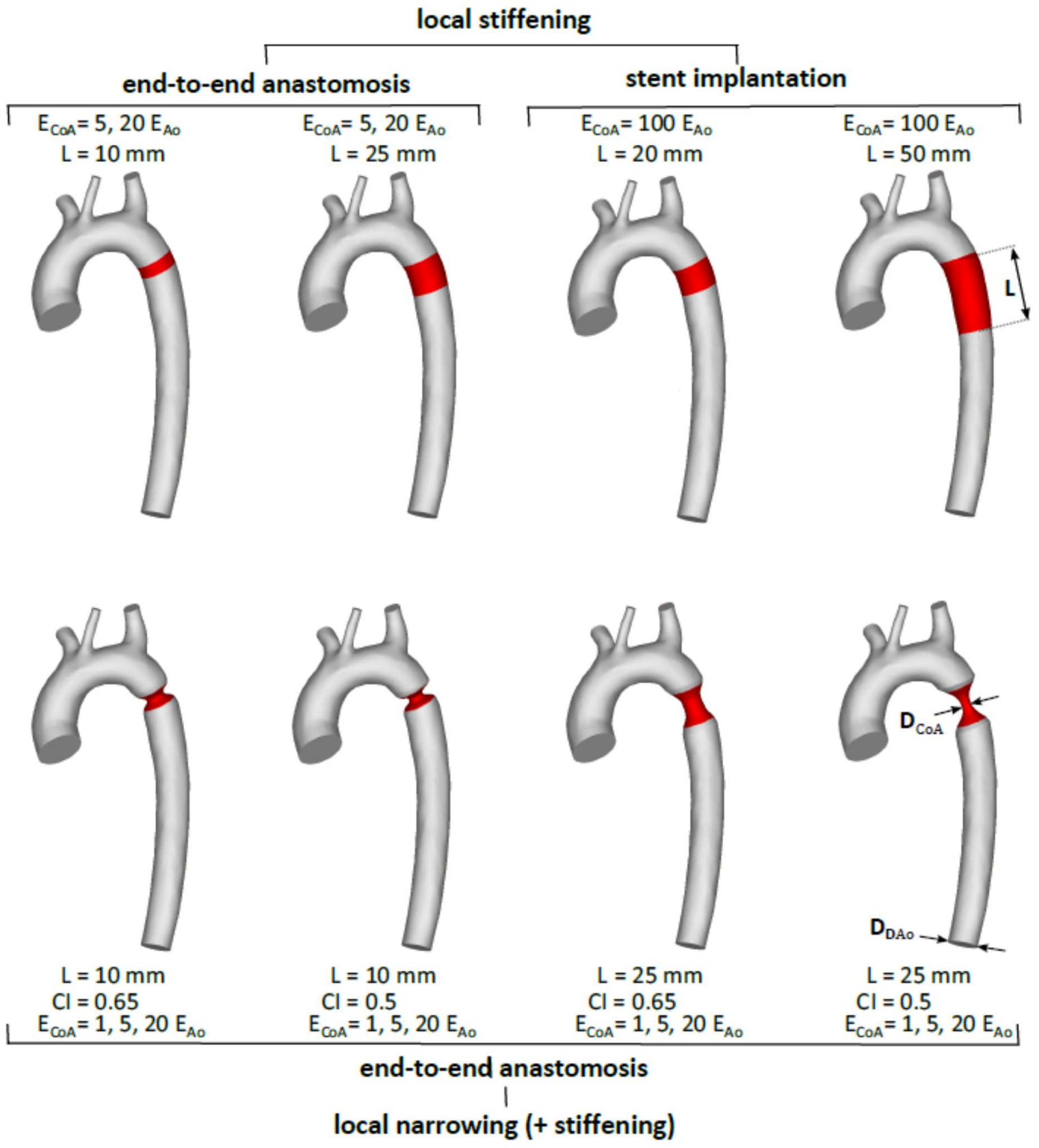

Fig. 2 

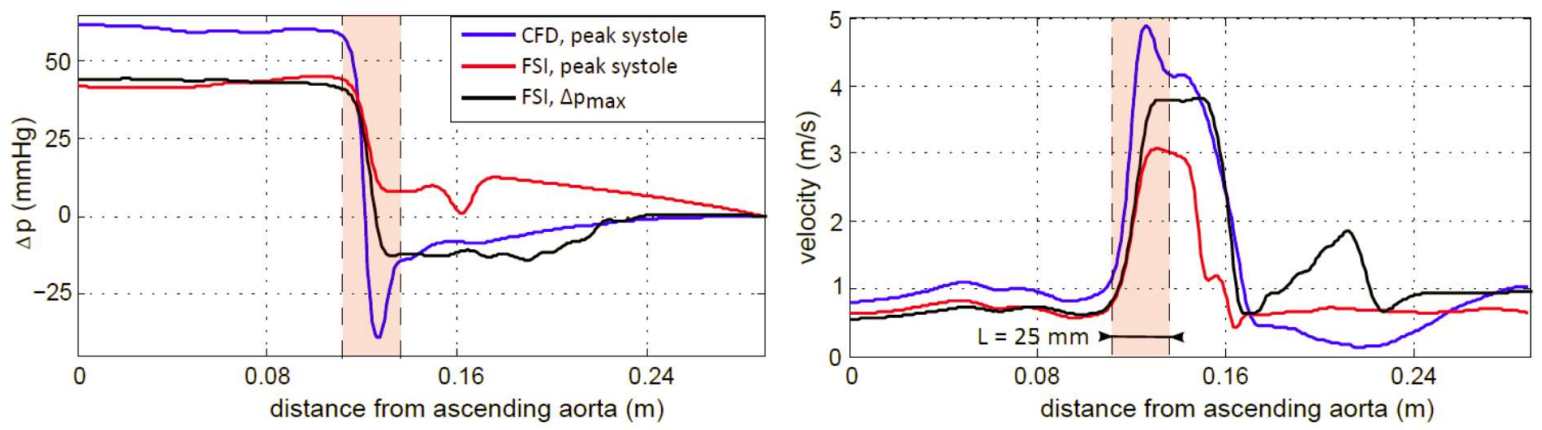

Fig. 3 

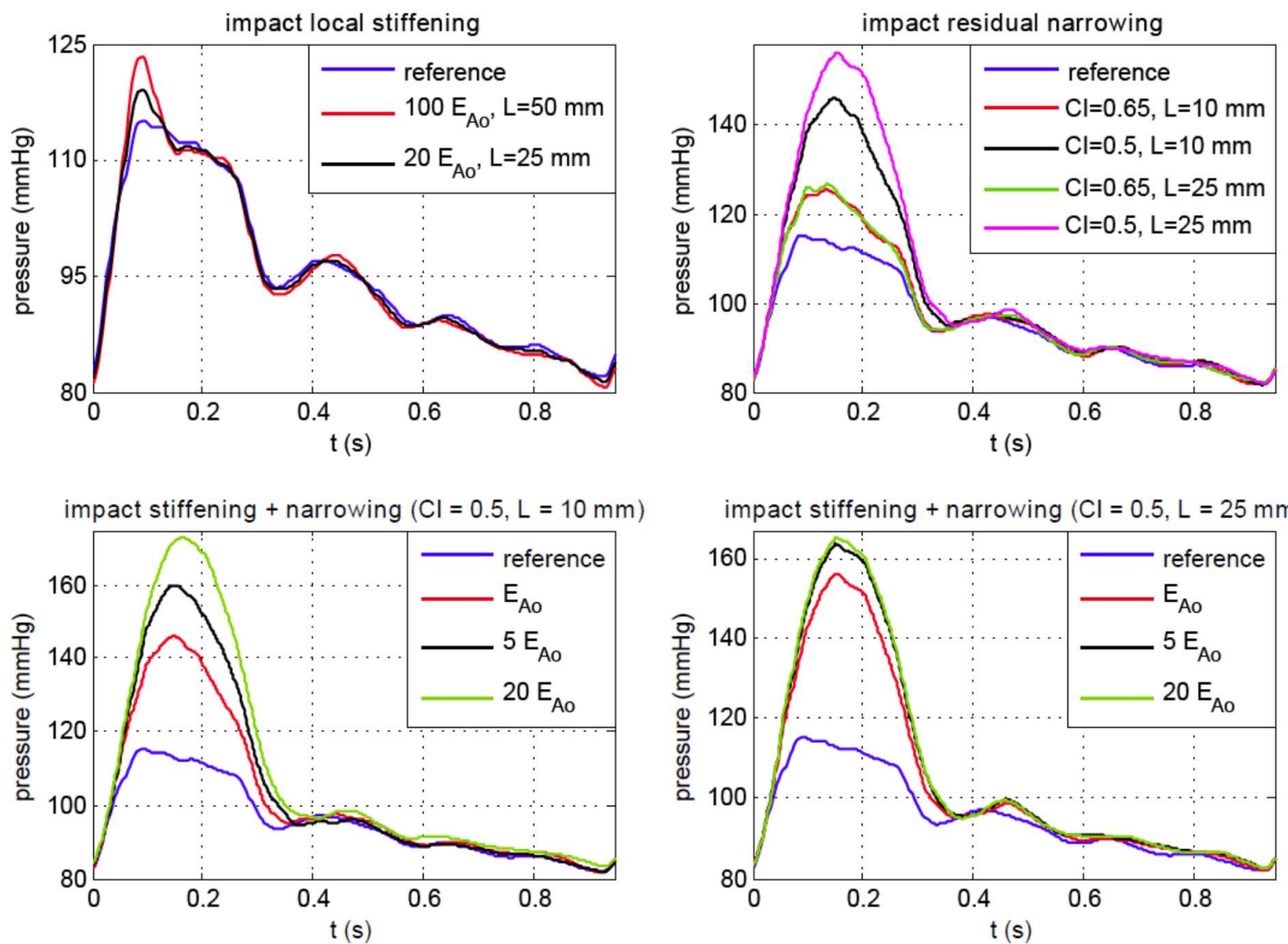

Fig. 4 

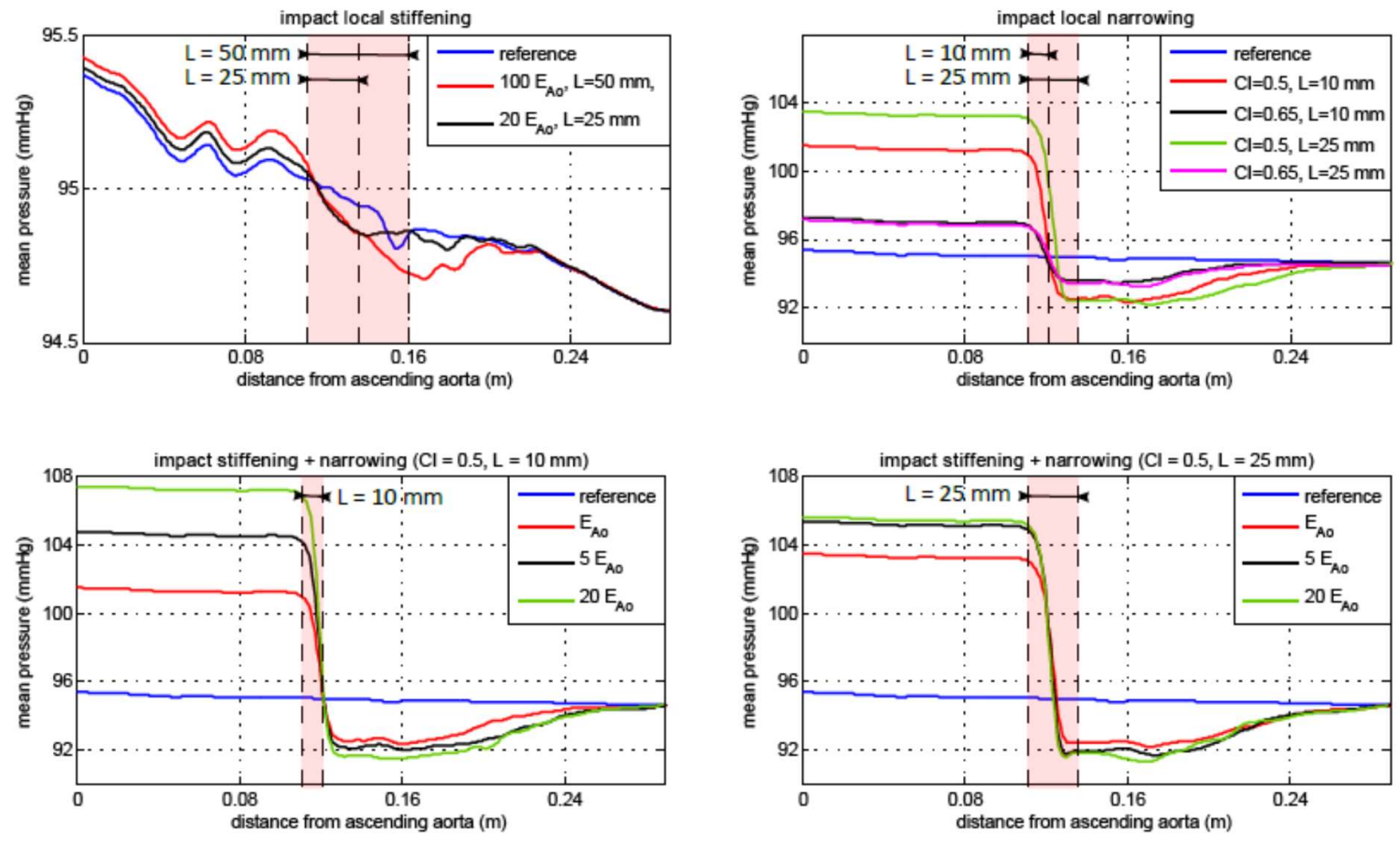

Fig. 5 

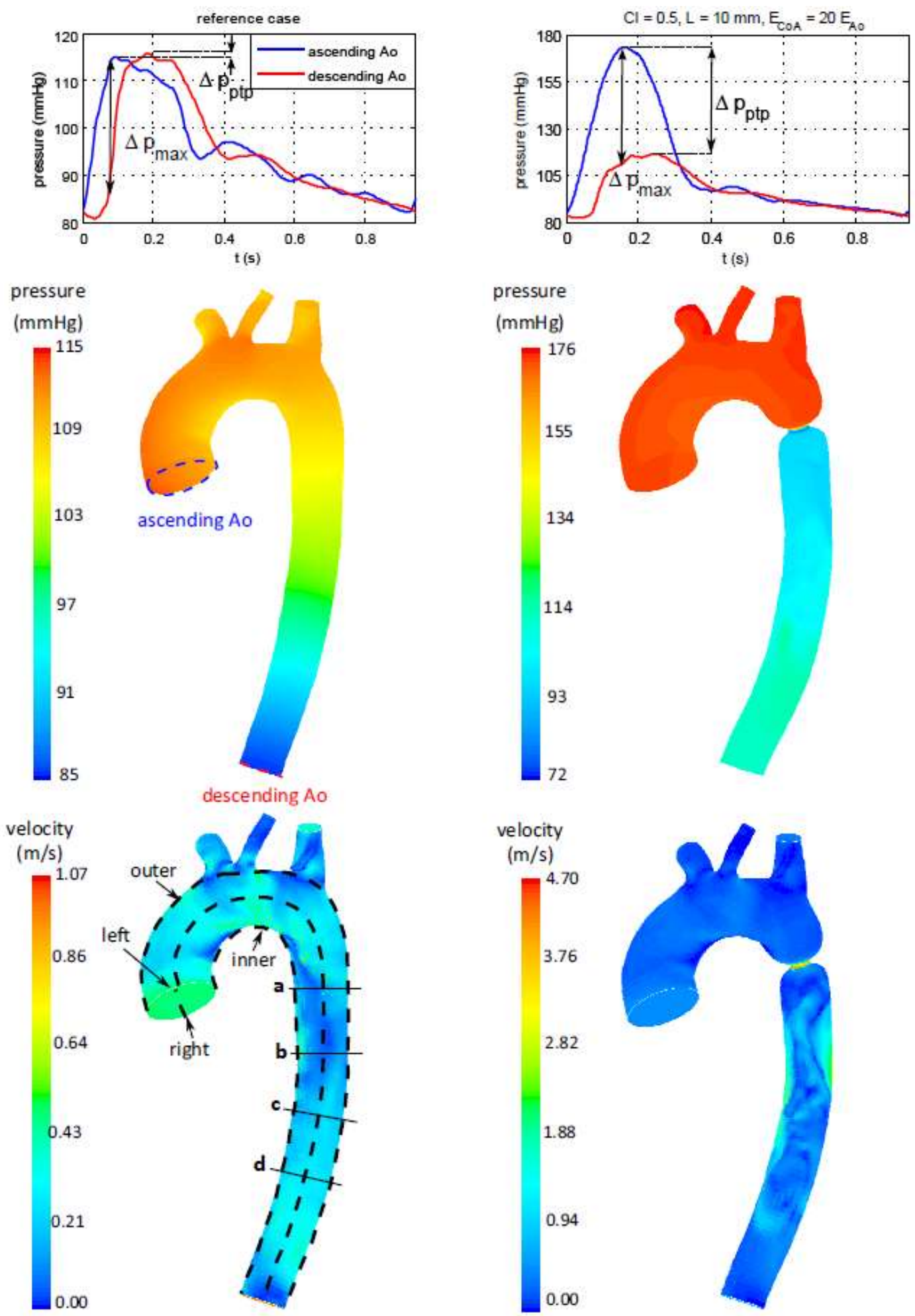

Fig. 6 


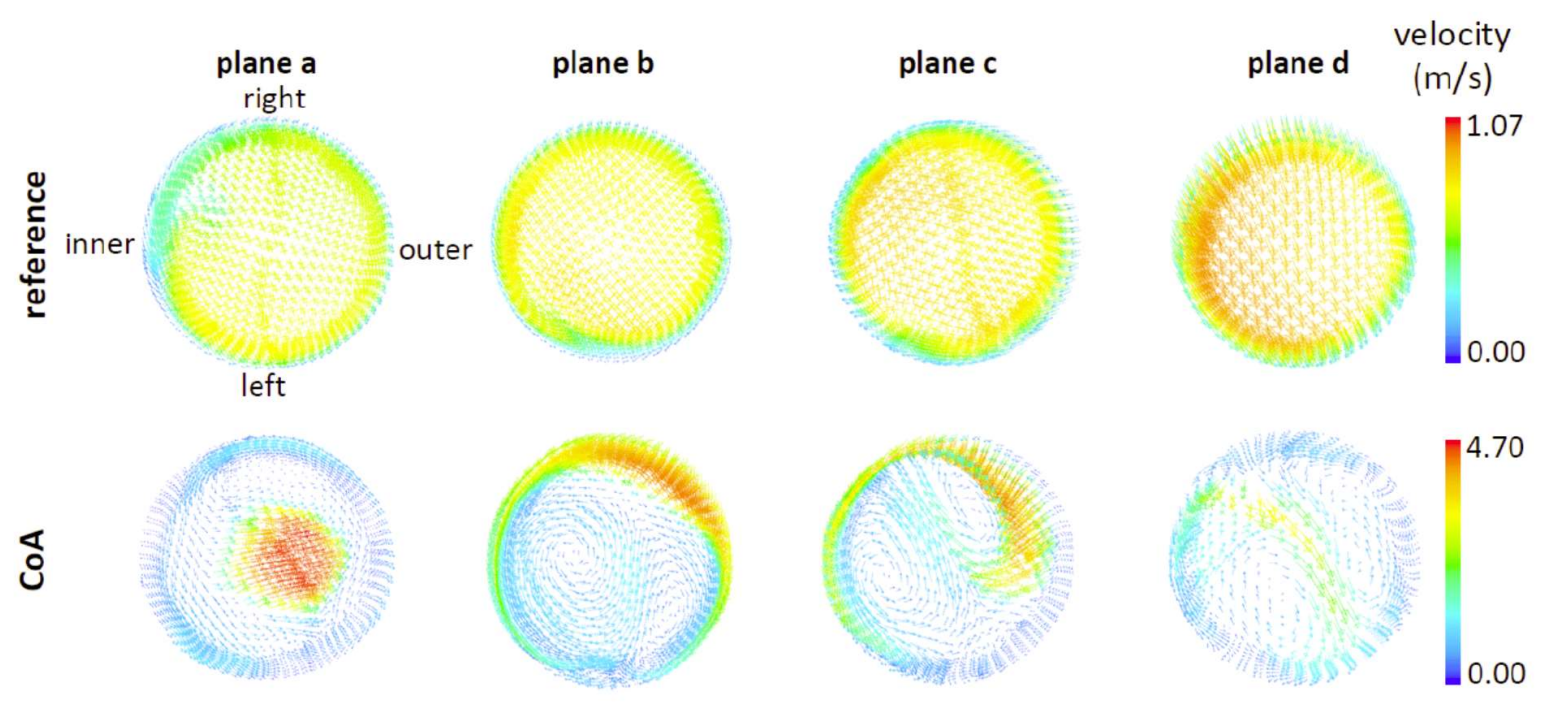

Fig. 7 
Appendix 1: Mesh and time step sensitivity study

Given the focus of the study on pressure, the criterion for the grid convergence and time step dependency study was the accuracy of the predicted pressures along the aortic arch. The case used for the analysis was the case with the shortest stenosis length $(1 \mathrm{~cm})$ and highest degree of stenosis (coarctation index 0.5).

\section{A1.1. Mesh sensitivity analysis}

Four different, full hexahedral uniform meshes (R1, R2, R3, R4) with an increasing number of elements in the boundary layer (ranging from 4 to 8 layers), the transversal and axial direction were constructed, with R4 considered as reference. A conforming mesh was applied in the fluid and solid domain. The number of cells is depicted in Table 1, together with the calculation time required to compute one cardiac cycle (on two 10-core Intel Xeon E5-2680v2 processors).

\begin{tabular}{|c|c|c|c|c|c|c|c|c|c|c|c|}
\hline \multirow{2}{*}{ Grid } & \multirow{2}{*}{$\begin{array}{l}\text { \#Fluid } \\
\text { cells }\end{array}$} & \multirow{2}{*}{$\begin{array}{l}\text { \#Solid } \\
\text { elements }\end{array}$} & \multirow{2}{*}{$\begin{array}{c}\text { Calc. } \\
\text { time/cycle }\end{array}$} & \multicolumn{8}{|c|}{ Mean error $(\%)$} \\
\hline & & & & asc & p1 & p2 & coa & $\mathrm{d} 1$ & $\mathrm{~d} 2$ & $\mathrm{~d} 3$ & desc \\
\hline R1 & $42 k$ & $21 \mathrm{k}$ & $12 \mathrm{~h} 29 \mathrm{~min}$ & 1.44 & 1.42 & 1.42 & 1.72 & 1.61 & 1.56 & 1.33 & 0.77 \\
\hline R2 & $105 \mathrm{k}$ & $37 \mathrm{k}$ & 17h29min & 1.01 & 0.98 & 0.98 & 1.26 & 1.54 & 1.88 & 1.26 & 0.71 \\
\hline R3 & $281 \mathrm{k}$ & $74 \mathrm{k}$ & 30h55min & 0.38 & 0.34 & 0.34 & 0.75 & 1.22 & 1.07 & 0.94 & 0.64 \\
\hline $\mathrm{R} 4$ & $408 \mathrm{k}$ & $102 k$ & $40 \mathrm{~h} 38 \mathrm{~min}$ & \multicolumn{8}{|c|}{ Reference grid } \\
\hline R5 & $216 \mathrm{k}$ & $51 \mathrm{k}$ & $23 \mathrm{~h} 38 \mathrm{~min}$ & 0.5 & 0.47 & 0.47 & 0.86 & 1 & 1.15 & 0.98 & 0.6 \\
\hline
\end{tabular}

Table A1. Grid refinement study of the pressure in an FSI model of aortic coarctation 
As the flow distal to the stenosis is complex and highly disturbed (Reynolds numbers up to 11 139), a high mesh density is required in this region to resolve the flow field in space. This is realized by locally adapting the fluid mesh. The resulting fluid mesh (R5) has, compared to the finest mesh (R4), a higher mesh density in the coarctation zone, but a coarser grid proximal to the stenosis and in the lower part of the descending aorta (see Figure A1).

Figure A2 depicts the pressure along the centerline of the aorta at peak systole, with Table A1 tabulating the mean error of the pressure in different cross sections (indicated by the dashed lines in Figure A2). These errors are defined with respect to the reference grid R4 and relative to the pressure amplitude in the corresponding cross section. The mean error thereby denotes the error averaged over one cardiac cycle and over the respective cross section. From the results in Table 1, it can be seen that even for meshes with a low cell density, the mean errors proximal, halfway and distal to the coarctation zone remain low $(<2 \%)$. When comparing the locally refined grid $\mathrm{R} 5$ with the uniformly refined grids $\mathrm{R} 3$ and $\mathrm{R} 4$, an important reduction in computation time is gained ( 23 h 38 min per cardiac cycle versus 30 h 33 min and 40 h 38 min) without a loss in accuracy. The mean error obtained with the mesh R5 stays below 1.15\% and comparable errors are found as for the mesh R3. 

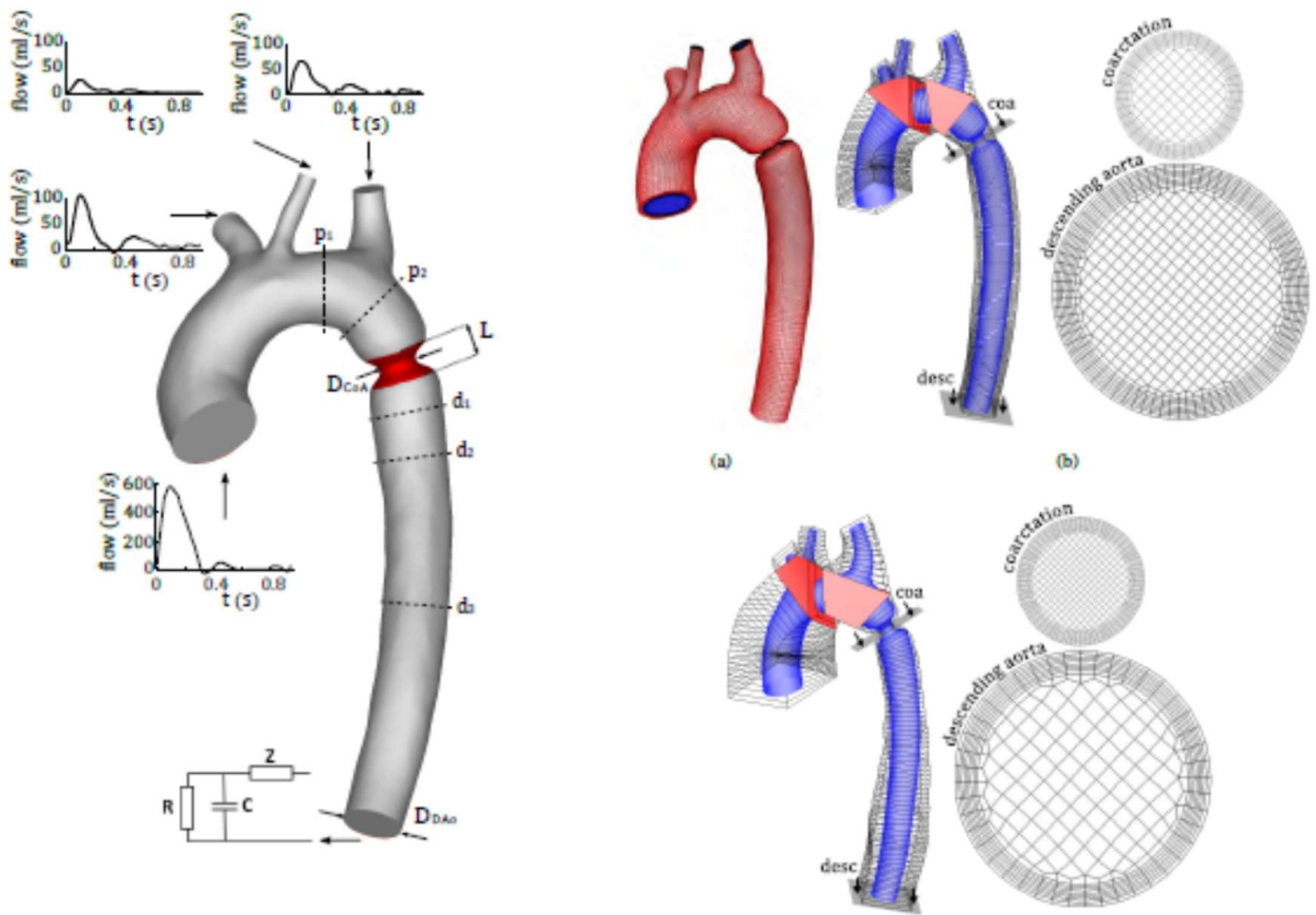

(c)

Figure A1. Left: indication of sections where pressure was calculated (p: proximal; d: distal). Right a) Mesh for the fluid domain (blue) and the arterial wall (red) of an aortic arch with aortic coarctation. Note the axial coarsening towards the descending aorta (R5). (b) and (c) The cross sectional grids of the fluid mesh at the coarctation (coa) and the descending aorta (desc), which result from multiblock structures R4 (uniform grid refinement) and R5 (local grid refinement). 


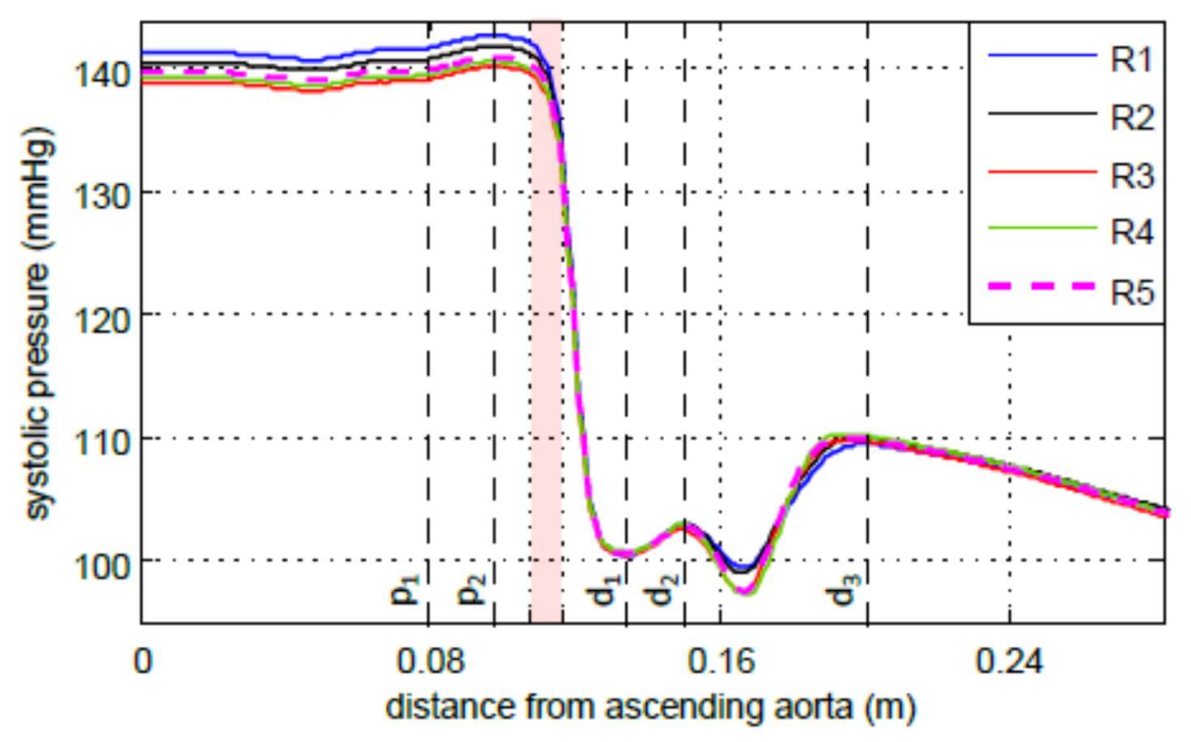

Figure A2. Pressure along the centerline at peak systole for increasing mesh densities (R1 to R4) and a grid with a local refinement at the coarctation region and a gradual coarsening towards the descending aorta (R5).

\section{A1.2. Time step sensitivity analysis}

Figure A3 illustrates the impact of the time step size on the pressure evolution at different cross sections along the aorta. It can be observed that the results in the proximal part and at the coarctation zone are more or less time step independent, whereas the small pressure oscillations in the distal part are not captured with a large time step size (of 4 or $5 \mathrm{~ms}$ ). Moreover, the oscillations developing in $\mathrm{d} 3$ are not even resolved properly with a time step size of $1 \mathrm{~ms}$. As such, the time step size was further decreased to 0.5 and $0.25 \mathrm{~ms}$ and the results are shown in Figure A4. An unstable behavior was found if a small time step size was applied. The observed oscillations responsible for this behavior were indeed not resolved for the simulations using larger time step sizes. Because the oscillations itself are resolved by multiple time steps and the frequency of the oscillations is more or less time step independent, it is presumed that these 
oscillations do not arise from a numerical instability but have a physical origin, triggered by the disturbed blood flow. In a physiological setting, this oscillation would however be cushioned by the damping nature of the surrounding tissue. We believe that the lack of physical damping in our model resulted in the observed oscillations that eventually got unstable when using a time step size smaller than $1 \mathrm{~ms}$.

To test this hypothesis, Rayleigh damping was added to the structural model and the simulation using a time step size of $0.5 \mathrm{~ms}$ was repeated. The Rayleigh damping coefficients $\alpha$ and $\beta$ were selected such that $1 \%$ damping of the waves with a $1 \mathrm{~Hz}$ frequency (close to the frequency of the cardiac cycle) was obtained and $20 \%$ damping for the $250 \mathrm{~Hz}$ waves (i.e. the frequency of the observed oscillations). These constrictions resulted in a value of 0.116 for the mass proportional damping parameter $\alpha$ and 0.000255 for the stiffness proportional damping $\beta$. It is demonstrated in Figure A4 that the unstable behavior indeed disappears with the use of Rayleigh damping.

The larger pressure oscillations at the start of the simulation $(\mathrm{t}<0.2 \mathrm{~s})$ for the case with Rayleigh damping is explained by the temporal discretization schemes used at the start. The simulation without damping is started with a first-order scheme, to facilitate the startup. After $0.2 \mathrm{~s}$ the accuracy is improved by switching to a second-order scheme. For the case with damping, a second-order scheme can be used from the start on. In this study, a time step size of $2 \mathrm{~ms}$ has been used as a compromise between accuracy and computation time. The error obtained with this time step size is sufficiently smaller than the mutual differences in results. 

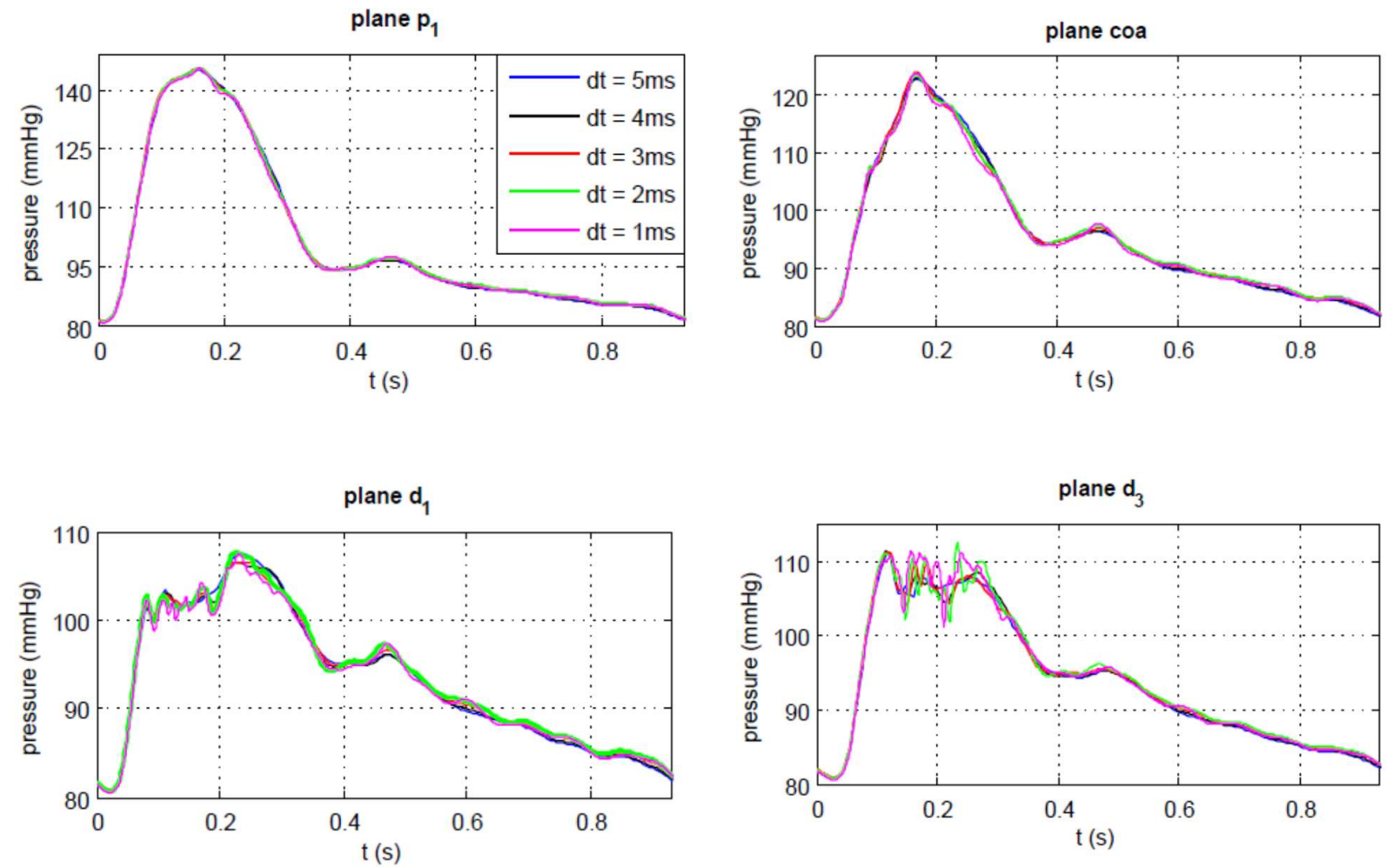

Figure A3. Influence of the time step size on the pressure evolution at proximal cross section $\mathrm{p} 1$, halfway the coarctation zone (coa) and at two distal cross sections (d1 and d3). See Figure A1 for an indication of these plane locations. 

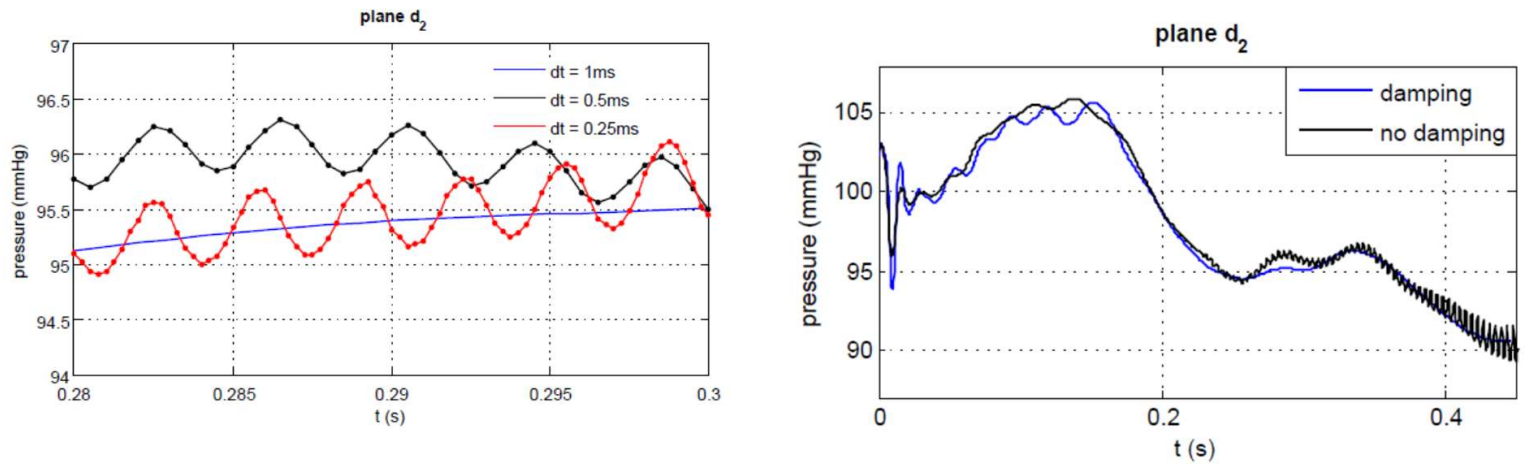

Figure A4. Left: Detail of the pressure evolution, illustrating the temporal resolution of the oscillations.Right: Influence of Rayleigh damping on the pressure evolution at cross section $\mathrm{d} 2$. Inclusion of Rayleigh damping prevents the simulation from unstable behavior. 